Editor's Note: These short reviews of recent JNeurosci articles, written exclusively by students or postdoctoral fellows, summarize the important findings of the paper and provide additional insight and commentary. If the authors of the highlighted article have written a response to the Journal Club, the response can be found by viewing the Journal Club at www.jneurosci.org. For more information on the format, review process, and purpose of Journal Club articles, please see http://jneurosci.org/content/ preparing-manuscript\#journalclub.

\title{
The Role of Neuroligins in the Cerebellum Highlights the Diversity of Synapse-Specifying Molecules
}

\author{
- Dessica Messier \\ Department of Neuroscience and Medical Scientist Training Program, Baylor College of Medicine, Houston, Texas 77030, and The Cain Foundation \\ Laboratories, Jan and Dan Duncan Neurological Research Institute, Texas Children's Hospital, Houston, Texas 77030 \\ Review of Zhang and Südhof
}

The brain integrates and processes sensory input to generate outputs that determine our actions, emotions, and thoughts. This impressive task is accomplished by transmitting signals through synapses between neurons, which are interconnected in a highly specific pattern. During the process of synapse formation, there first is establishment of synaptic contacts, followed by the assembly of specific presynaptic and postsynaptic molecular machinery and functional specification to confer specific properties to the synapse. Functional specification determines the extent to which the postsynaptic receptors will respond to the neurotransmitters released from the presynaptic cell. Synaptic cell adhesion molecules are a family of proteins that contribute to synapse specification. Different synaptic adhesion molecules located on the presynaptic and postsynaptic cells can interact in a heterophilic manner through their extracellular domains and then can transmit transsynaptic signals intracellularly through their cytoplasmic tails to recruit different presynaptic and postsynaptic molecular machineries, thereby contributing to the functional properties of that synapse (Missler et al.,

Received Oct. 26, 2016; revised Dec. 10, 2016; accepted Dec. 12, 2016.

J.M. is a McNair Student Scholar at Baylor College of Medicine. I thank Dr. Mingshan Xue for his continued support, comments, and discussions.

Correspondence should be addressed to Jessica Messier, 1250 Moursund Street, Suite 1250, Houston, TX 77030. E-mail: messier@bcm.edu. DOI:10.1523/JNEUROSCI.3317-16.2017

Copyright $\odot 2017$ the authors $\quad 0270-6474 / 17 / 370739-03 \$ 15.00 / 0$
2012). Many families of cell adhesion molecules, including postsynaptic neuroligins, cerebellins, calsyntenins, and leucine-rich repeat transmembrane neuronal proteins (LRRTMs), which can all bind to presynaptic neurexins to specify synapses, are involved in synapse formation. Many of these molecules can undergo alternative splicing, leading to altered binding affinities to other adhesion molecules, furthering adding to their diversity (de Wit and Ghosh, 2016). The large diversity of synaptic cell adhesion molecules suggests that they may provide a trans-synaptic code that can differentially participate in synapse specification depending on the brain region and/or cell types, which could explain the diversity of synapses found in the brain. However, the extent to which synapse-specifying molecules act across different brain regions and synapses to establish connectivity and regulate network properties is incompletely understood.

Of the synaptic cell adhesion molecules, neuroligins are probably the most studied because they are associated with various neuropsychiatric disorders, including autism and schizophrenia (Südhof, 2008). Neuroligins are a class of four postsynaptic cell adhesion molecules (Nlgn1-Nlgn4) that are localized to the postsynaptic membrane of glutamatergic (Nlgn1 and Nlgn3), GABAergic (Nlgn2 and Nlgn3), cholinergic (Nlgn2), or glycinergic (Nlgn4) synapses (Hu et al., 2015). Recent work suggests that Nlgn1 regulates NMDAR- mediated excitation in the cortex, hippocampus, and amygdala; Nlgn 2 regulates inhibition in the hippocampus, dentate gyrus, brainstem, and cortex, and, at least in the somatosensory cortex, is specific to parvalbumin $(\mathrm{Pv})$ expressing interneuron-mediated inhibition; and Nlgn3 regulates excitation in the hippocampus and cerebellum, but not in the somatosensory cortex or dorsal striatum (Hu et al., 2015). In addition, Nlgn3 enhances inhibition onto $\mathrm{D}_{1}$, but not $\mathrm{D}_{2}$ dopamine receptorexpressing medium spiny neurons (MSNs) of the dorsal striatum (Rothwell et al., 2014), whereas it acts to attenuate inhibition mediated by cholecystokinin-expressing basket cells, but not Pv-expressing cells, in hippocampal pyramidal cells (Földy et al., 2013). These results suggest that neuroligins might regulate synaptic specification and function in a circuit- and synapsespecific manner.

To further unravel the extent to which synapse-specifying molecules act across different brain regions and synapses, Zhang and Südhof (2016) investigated the role of neuroligins specifically in cerebellar stellate cells. These cells are GABAergic interneurons that receive direct excitation from parallel fibers, but not climbing fibers, and inhibition from stellate cells and other interneurons. The authors used mouse lines in which the expression of any of the three major neuroligins present in the cerebellum (Nlgn1, Nlgn2, and Nlgn3) can be eliminated in cells expressing Cre recombinase. 
They crossed these mice with $P v$-Cre mice to remove neuroligins from Purkinje cells, stellate cells, and other interneurons in the cerebellum ( $\mathrm{Pv}-\mathrm{Nlgn} 123 \mathrm{cKO})$. While recording from stellate cells in an acute slice preparation, the authors stimulated the nearby molecular layer of the cerebellum to activate parallel fibers and stellate cells. They pharmacologically blocked inhibitory or excitatory synaptic transmission to measure the EPSCs from parallel fibers or IPSCs from stellate cells, respectively.

Neuroligin-deficient cerebellar stellate cells had a large reduction in glutamatergic EPSCs mediated by NMDARs postsynaptic to parallel fiber inputs, but there was no change in AMPAR-mediated EPSCs at these synapses (Zhang and Südhof, 2016). This same phenotype was seen in stellate cells lacking only Nlgn1. This result is interesting because NMDARs in stellate interneurons are expressed perisynaptically rather than within the synaptic density (Clark and CullCandy, 2002), and thus this result indicates that, in addition to regulating synaptic NMDAR-mediated EPSCs in the cortex, hippocampus, and amygdala ( $\mathrm{Hu}$ et al., 2015), Nlgn1 can also regulate extrasynaptic NMDAR-mediated EPSCs in cerebellar stellate cells. Whether Nlgn1 interacts directly or indirectly with extrasynaptic NMDARs is unknown, however. A next step might be to investigate the association of Nlgn1 with G- $\alpha$-interacting proteininteracting protein, $\mathrm{C}$ terminus, a PDZ protein that is preferentially associated with extrasynaptic NMDARs in cultured hippocampal neurons (Yi et al., 2007).

The role of neuroligins in cerebellar stellate cells differs from their role in Purkinje cells. In a previous study, Zhang et al. (2015) showed that Purkinje cells lacking Nlgn1, Nlgn2, and Nlgn3; both Nlgn1 and Nlgn3; Nlgn1 alone; or Nlgn3 alone all had a significant reduction in EPSCs mediated by AMPARs at climbing fiber synapses, with no change in EPSCs at parallel fiber synapses. Since neuroligins do not regulate AMPARmediated excitation from parallel fibers onto Purkinje cells or stellate cells, then what molecules do? It is possible that cerebellin-1 is involved in this role. It is known that cerebellin-1 is secreted by parallel fibers and is essential for the formation of parallel fiber-Purkinje cell synapses by mediating the interaction between presynaptic neurexins on parallel fibers and postsynaptic Glu $\delta 2$ on Purkinje cells (Uemura et al., 2010). In addition, Glu $\delta 1$ is required for the formation of parallel fiber-stellate cell synapses (Konno et al., 2014). Although the role of cerebellin-1 in regulating parallel fiberstellate cell synapses is unknown, it is possible that cerebellin-1 regulates AMPARmediated excitation from parallel fiber synapses onto stellate cells through Glu $\delta 1$ in a manner that is similar to how it regulates parallel fiber-Purkinje cell synapses through Glu $\delta 2$, explaining the lack of an AMPAR synaptic phenotype observed in neuroligin-deficient stellate cells. However, it is unknown whether deletion of Nlgn1, Nlgn2, and Nlgn3 from cerebellar stellate cells, during early development, as performed in the study by Zhang and Südhof (2016), produces no parallel fiber AMPARmediated synaptic phenotype because another cell adhesion molecule, potentially cerebellin-1, normally has this role or because it is assuming a compensatory role. A possible compensatory role of cerebellin-1 is supported by recent work (Zhang et al., 2016) showing that the deletion of Nlgn3 from the calyx of Held during early development results in no detectable phenotype due to compensation from cerebellin-1, whereas the deletion of Nlgn3 during late development produces a strong synaptic phenotype. To test whether compensation is occurring, Nlgn1, Nlgn2, and Nlgn3 should be deleted from cerebellar stellate cells at a later time point in development.

Zhang and Südhof (2016) also showed that neuroligin-deficient cerebellar stellate cells had a modest $(\sim 20 \%)$ reduction in miniature IPSC (mIPSC) amplitude. Although they did not investigate which neuroligin subtype was responsible for the modest reduction in inhibition, it is most likely attributable to Nlgn2, which is required for proper stellate and basket cell-mediated inhibition of Purkinje cells (Zhang et al., 2015), for proper inhibition of CA1 hippocampal pyramidal cells (Poulopoulos et al., 2009), and for Pv cell-mediated inhibition of cortical pyramidal cells (Gibson et al., 2009). The question that remains is why do stellate cells lacking Nlgn1, Nlgn2, and Nlgn3 have only a modest reduction in stellate cell-mediated inhibition when, in the same neural circuit, Purkinje cells lacking Nlgn1, Nlgn2, and Nlgn3 have a large $45 \%$ and $80 \%$ reduction in mIPSC amplitude and frequency, respectively, from the same presynaptic stellate cells (Zhang et al., 2015). A similar phenomenon has been observed with Nlgn3 deletion in MSNs of the dorsal striatum, where the deletion of Nlgn3 from MSNs expressing $\mathrm{D}_{1}$ dopamine receptors $\left(\mathrm{D}_{1}-\mathrm{MSN}\right)$ reduces inhibition in these cells, whereas the deletion of Nlgn3 in MSNs expressing $\mathrm{D}_{2}$ dopamine receptors $\left(\mathrm{D}_{2}\right.$-MSN) does not affect inhibition (Rothwell et al., 2014). One possibility is that the stellate-stellate synapse in the cerebellum and the inhibitory synapse onto $\mathrm{D}_{2}$-MSNs normally use, or are able to compensate with, trans-synaptic molecules that are not Nlgn 1, Nlgn2, or Nlgn3, whereas the stellate-Purkinje cell synapse and the inhibitory synapse onto $\mathrm{D}_{1}$-MSNs do not have this ability. Since LRRTMs, calsyntenins, and cerebellins are also able to bind to presynaptic neurexins to specify synapses (de Wit and Ghosh, 2016), it is potentially one of these postsynaptic cell adhesion molecules that is partially responsible for regulating the stellate-stellate inhibitory synapse.

Another potential explanation for the modest reduction in inhibition onto neuroligin-deficient stellate cells is that the deletion of Nlgn 2 or Nlgn3 alone would have opposite effects on IPSCs. As previously mentioned, Nlgn2 deletion alone will most likely decrease inhibition in stellate cells. In contrast, Nlgn3 deletion might result in increased inhibition onto stellate cells by reducing tonic endocannabinoid signaling, thus increasing the probability of GABA release, because such an effect was reported for hippocampal pyramidal neurons lacking Nlgn3 (Földy et al., 2013). Cerebellar stellate cells express type 1 endocannabinoid receptors (CB1Rs) on their presynaptic terminals (Ashton et al., 2004), and stellate cells can release endocannabinoids to activate presynaptic CB1Rs on parallel fibers to attenuate synaptic transmission (Beierlein and Regehr, 2006). Thus, it is possible that stellate cells can release endocannabinoids to activate CB1Rs on presynaptic stellate cell synaptic terminals, resulting in a reduction in GABA release probability at the stellatestellate synapse. The loss of Nlgn3 could decrease tonic endocannabinoid release from neuroligin-deficient stellate cells, resulting in enhanced GABA release probability, and thus larger IPSCs, from stellate cells onto neighboring stellate cells. Consequently, when both Nlgn 2 and Nlgn 3 are deleted simultaneously, the decreased and increased inhibition, respectively, result in a net modest reduction in the inhibition seen in these cells. This hypothesis can be tested in Nlgn3-null stellate cells. Nevertheless, the result of a modest reduction in inhibition seen in neuroligin-deficient stellate cells would further support the notion that different neuroligins play distinct roles in individual synapses and circuits.

In conclusion, the study by Zhang and Südhof (2016) not only revealed that Nlgn1 regulates extrasynaptic NMDAR-mediated excitation at the parallel fiber synapse onto cerebellar stellate cells, but also further highlighted the diversity of synapse-specifying molecules across different brain regions and synapse types. Future studies should aim to 
uncover the role of neuroligins in other cell types and synapses, as well as to investigate other cell adhesion molecules, such as LRRTMs, calsyntenins, and cerebellins, in various neural circuits. This knowledge will provide insight into how neural circuits are assembled in healthy brains, as well as how neural circuits might be altered in neuropsychiatric disorders.

\section{References}

Ashton JC, Appleton I, Darlington CL, Smith PF (2004) Immunohistochemical localization of cannabinoid CB1 receptor in inhibitory interneurons in the cerebellum. Cerebellum 3:222-226. CrossRef Medline

Beierlein M, Regehr WG (2006) Local interneurons regulate synaptic strength by retrograde release of endocannabinoids. J Neurosci 26: 9935-9943. CrossRef Medline

Clark BA, Cull-Candy SG (2002) Activity-dependent recruitment of extrasynaptic NMDA receptor activation at an AMPA receptor-only synapse. J Neurosci 22:4428-4436. Medline

de Wit J, Ghosh A (2016) Specification of synaptic connectivity by cell surface interactions. Nat Rev Neurosci 17:22-35. CrossRef Medline

Földy C, Malenka RC, Südhof TC (2013) Autismassociated neuroligin-3 mutations commonly disrupt tonic endocannabinoid signaling. Neuron 78:498-509. CrossRef Medline
Gibson JR, Huber KM, Südhof TC (2009) Neuroligin-2 deletion selectively decreases inhibitory synaptic transmission originating from fast-spiking but not from somatostatinpositive interneurons. J Neurosci 29:1388313897. CrossRef Medline

Hu X, Luo JH, Xu J (2015) The interplay between synaptic activity and neuroligin function in the CNS. Biomed Res Int 2015:498957. CrossRef Medline

Konno K, Matsuda K, Nakamoto C, Uchigashima M, Miyazaki T, Yamasaki M, Sakimura K, Yuzaki M, Watanabe M (2014) Enriched expression of GluD1 in higher brain regions and its involvement in parallel fiber-interneuron synapse formation in the cerebellum. J Neurosci 34:7412-7424. CrossRef Medline

Missler M, SüdhofTC, Biederer T (2012) Synaptic cell adhesion molecules. Cold Spring Harb Perspect Biol 4:a005694. CrossRef Medline

Poulopoulos A, Aramuni G, Meyer G, Soykan T, Hoon M, Papadopoulos T, Zhang M, Paarmann I, Fuchs C, Harvey K, Jedlicka P, Schwarzacher SW, Betz H, Harvey RJ, Brose N, Zhang W, Varoqueaux F (2009) Neuroligin 2 drives postsynaptic assembly at perisomatic inhibitory synapses through gephyrin and collybistin. Neuron 63:628-642. CrossRef Medline

Rothwell PE, Fuccillo MV, Maxeiner S, Hayton SJ, Gokce O, Lim BK, Fowler SC, Malenka RC, Südhof TC (2014) Autism-associated neuroligin-3 mutations commonly impair striatal circuits to boost repetitive behaviors. Cell 158:198-212. CrossRef Medline

Südhof TC (2008) Neuroligins and neurexins link synaptic function to cognitive disease. Nature 455:903-911. CrossRef Medline

Uemura T, Lee SJ, Yasumura M, Takeuchi T, Yoshida T, Ra M, Taguchi R, Sakimura K, Mishina M (2010) Trans-synaptic interaction of GluRD2 and neurexin through Cbln1 mediates synapse formation in the cerebellum. Cell 141:1068-1079. CrossRef Medline

Yi Z, Petralia RS, Fu Z, Swanwick CC, Wang YX, Prybylowski K, Sans N, Vicini S, Wenthold RJ (2007) The role of the PDZ protein GIPC in regulating NMDA receptor trafficking. J Neurosci 27:11663-11675. CrossRef Medline

Zhang B, Südhof TC (2016) Neuroligins are selectively essential for NMDAR signaling in cerebellar stellate interneurons. J Neurosci 36: 9070-9083. CrossRef Medline

Zhang B, Chen LY, Liu X, Maxeiner S, Lee SJ, Gokce O, Südhof TC (2015) Neuroligins sculpt cerebellar Purkinje-cell circuits by differential control of distinct classes of synapses. Neuron 87:781-796. CrossRef Medline

Zhang B, Seigneur E, Wei P, Gokce O, Morgan J, Südhof T (2016) Developmental plasticity shapes synaptic phenotypes of autismassociated neuroligin- 3 mutations in the calyx of Held. Mol Psychiatry. Advance online publication. Retrieved December 15, 2016. doi:10.1038/mp.2016.157. CrossRef Medline 